\title{
SIGN-CHANGING SOLUTIONS FOR A CLASS OF NONLINEAR SCHRÖDINGER EQUATIONS
}

\author{
XIANGQING LIU ${ }^{凶}$ and YISHENG HUANG
}

(Received 3 November 2008)

\begin{abstract}
Using variational methods, we obtain the existence of sign-changing solutions for a class of asymptotically linear Schrödinger equations with deepening potential well.
\end{abstract}

2000 Mathematics subject classification: primary 35J10; secondary 35J20, 35J60.

Keywords and phrases: Schrödinger equation, asymptotically linear, variational method, sign-changing solution.

\section{Introduction}

In this paper we are concerned with the following nonlinear Schrödinger equation

$$
\left\{\begin{array}{l}
-\Delta u+V_{\lambda}(x) u=f(x, u) \quad \text { in } \mathbb{R}^{N}, N \geq 3 \\
u \in H^{1}\left(\mathbb{R}^{N}\right),
\end{array}\right.
$$

where $V_{\lambda}(x)=1+\lambda g(x), \lambda$ is a positive parameter, the function $g$ satisfies the condition $(\mathrm{G})$ :

(G) $g \in L^{\infty}\left(\mathbb{R}^{N}, \mathbb{R}\right)$ and there exists a nonempty bounded smooth domain $\Omega \subset \mathbb{R}^{N}$ such that

$$
g(x) \equiv 0 \quad \text { on } \bar{\Omega}, \quad g(x) \in(0,1] \quad \text { on } \mathbb{R}^{N} \backslash \bar{\Omega} \quad \text { and } \quad \lim _{|x| \rightarrow \infty} g(x)=1 .
$$

We make the following assumptions on $f$ :

$\left(f_{1}\right) \quad f(x, t) \in C\left(\mathbb{R}^{N} \times \mathbb{R}, \mathbb{R}\right), f(x, t) t \geq 0$ for almost every $x \in \mathbb{R}^{N}$, for all $t \in \mathbb{R}$;

$\left(f_{2}\right) \lim _{|t| \rightarrow 0}(f(x, t) / t)=0$ uniformly with respect to $x \in \mathbb{R}^{N}$;

$\left(f_{3}\right)$ there exists $\alpha \in(0, \infty)$ such that $\lim _{|t| \rightarrow \infty}(f(x, t) / t)=1+\alpha$ uniformly with respect to $x \in \bar{B}_{R}$ for all $R>0$, and $f(x, t) / t$ is bounded on $\mathbb{R}^{N} \times(\mathbb{R} \backslash\{0\})$;

$\left(f_{4}\right) \lim _{|x| \rightarrow \infty} \sup _{|t| \leq r}(|f(x, t)| /|t|)=0$ for every $r>0$.

This work was supported by the Natural Science Foundation of China (No. 10571174), a grant from Jiangsu Education Committee of China (No. 08KJB110009) and the Foundation of Yunnan Education Committee of China (No. 08Y0144).

(C) 2009 Australian Mathematical Publishing Association Inc. 0004-9727/2009 \$16.00 
In $\left(f_{3}\right), B_{R}$ denotes the ball in $\mathbb{R}^{N}$ centered at zero with radius $R$. Let us remark that there exist functions satisfying the hypotheses $\left(f_{1}\right)-\left(f_{4}\right)$, for example,

$$
f(x, t)=\frac{(1+\alpha) e^{-|x|^{2}} t^{3}}{1+e^{-|x|^{2}} t^{2}} .
$$

Usually, if there exists some real number $l$ such that:

$\left(f_{3}^{\prime}\right) \lim _{|t| \rightarrow \infty}(f(x, t) / t)=l$ uniformly with respect to $x \in \mathbb{R}^{N}$,

then we call $f$ asymptotically linear at infinity. However, the condition ' $\lim _{|t| \rightarrow \infty}(f(x, t) / t)=1+\alpha$ uniformly with respect to $x \in \bar{B}_{R}$ for all $R>0$ ' in the condition $\left(f_{3}\right)$ is weaker than $\left(f_{3}^{\prime}\right)$, and we point out that the technical condition $\left(f_{4}\right)$ will be mainly employed to prove the Palais-Smale condition ((PS) condition) in this paper (see Lemma 2.1).

When $f$ is sublinear or superlinear at infinity, some existence results related to Problem (1.1) were obtained recently, see for example [1-4, 9] and the references therein. In this paper, we focus on the case of $f$ asymptotically linear at infinity.

Let $\Omega$ be given by $(\mathrm{G})$. We denote by $\xi_{1}$ the first eigenvalue of the following Dirichlet problem

$$
\begin{cases}-\Delta u=\xi u & \text { in } \Omega \\ u=0 & \text { on } \partial \Omega .\end{cases}
$$

We also denote by $\lambda=\Lambda(\alpha)$ the principal eigenvalue of the following eigenvalue problem

$$
-\Delta u-\alpha u+\lambda g(x) u=0, \quad u \in H^{1}\left(\mathbb{R}^{N}\right), \alpha>0 .
$$

It has been proved in [7] that $\Lambda(\alpha)$ always exists for any $\alpha \in\left(\Gamma, \xi_{1}\right)$ with

$$
\Gamma=\inf \left\{\int_{\mathbb{R}^{N}}|\nabla u|^{2} d x: u \in H^{1}\left(\mathbb{R}^{N}\right) \text { and } \int_{\mathbb{R}^{N}}(1-g) u^{2} d x=1\right\},
$$

and the $\Lambda(\alpha)$-eigenfunction $u_{\Lambda(\alpha)}$ is the only eigenfunction which does not change sign. Moreover, $\Lambda(\alpha)>\alpha$, which is the largest eigenvalue among all other eigenvalues of (1.2).

Let us separate the first quadrant of the $(\lambda, \alpha)$-plane into several parts (see Figure 1), then we see from recent papers $[8,10,11]$ that the existence and nonexistence of signed solutions of Problem (1.1) have been completely discussed for these regions by using different methods. We summarize those results in the following Table 1.

In the existence cases of the preceding table (that is, regions apart from III, IV and the positive $\lambda$-axis), it is not hard to show the existence of solutions which do not change sign.

It is natural to ask whether sign-changing solutions for Problem (1.1) exist or not for the existence cases mentioned above? To the best of our knowledge, in any regions of the first quadrant of the $(\lambda, \alpha)$-plane, the existence and nonexistence results of signchanging solutions of Problem (1.1) have not been obtained. 


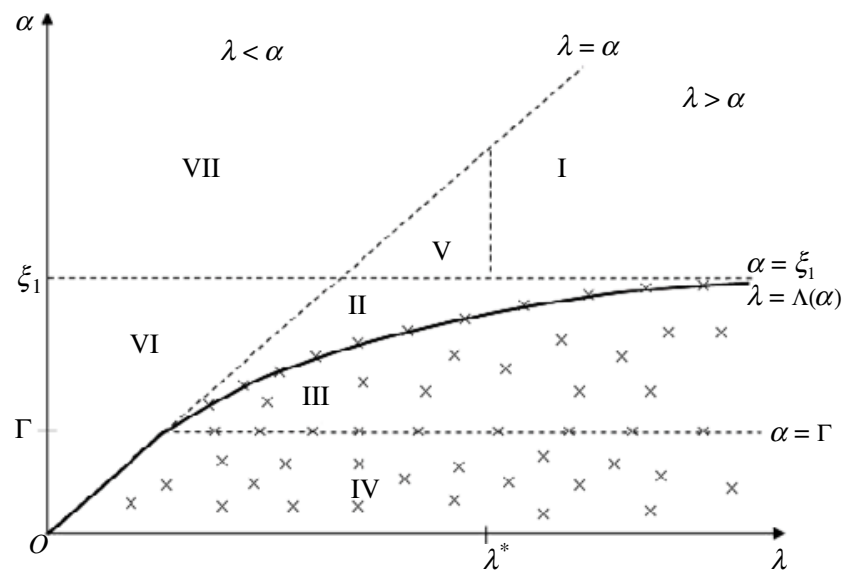

FIGURE 1. Separation of the first quadrant of the $(\lambda, \alpha)$-plane into several parts.

TABLE 1. Summary of results.

\begin{tabular}{llll}
\hline Relations for $\lambda$ and $\alpha$ & Corresponding regions & Obtained results & In papers \\
\hline$\alpha>\xi_{1}, \lambda>\alpha$ large & I & A positive solution & {$[10]$} \\
$\alpha \in\left(\Gamma, \xi_{1}\right), \lambda \in(\alpha, \Lambda(\alpha))$ & II & $\begin{array}{l}\text { A positive solution and } \\
\text { A negative solution }\end{array}$ & {$[8]$} \\
$\alpha \in\left(\Gamma, \xi_{1}\right), \lambda \geq \Lambda(\alpha)$ & III & No positive solution & {$[8]$} \\
$\alpha \leq \Gamma, \lambda \geq \alpha$ & IV & No positive solution & {$[8]$} \\
$\alpha=0, \lambda \in[0,+\infty)$ & The positive $\lambda$-axis & No positive solution & {$[11]$} \\
$\alpha>0, \lambda=0$ & The positive $\alpha$-axis & A positive solution & {$[11]$} \\
$\alpha>\Gamma, \lambda=\alpha$ & The dotted diagonal line & A positive solution & {$[11]$} \\
$\alpha=\xi_{1}, \lambda \in(\alpha,+\infty)$ & The dotted horizontal line & A positive solution & {$[11]$} \\
$\alpha>\xi_{1}, \lambda>0$ & I, V and VII & A positive solution & {$[11]$} \\
$\alpha>0, \lambda \in(0, \alpha)$ & VI and VII & A positive solution & {$[11]$} \\
\hline
\end{tabular}

In order to obtain sign-changing solutions of Problem (1.1), let us denote the operator $L_{\lambda}:=-\Delta+\lambda g(x), \Phi(u)=\int_{\mathbb{R}^{N}}\left(|\nabla u|^{2}+\lambda g(x) u^{2}\right) d x$, and let $\lambda$ be fixed in (1.2), then it follows from [6,7] that the lowest eigenvalue of $L_{\lambda}$ given by

$$
\alpha_{1}(\lambda)=\inf \left\{\Phi(u): u \in H^{1}\left(\mathbb{R}^{N}\right) \text { and }\|u\|_{2}=1\right\}
$$

is well defined, and there exists a positive eigenfunction $u_{\alpha_{1}(\lambda)}$ corresponding to $\alpha_{1}(\lambda)$. Moreover, $\alpha_{1}(\lambda)$ is simple in the sense of $\operatorname{ker}\left(L_{\lambda}-\alpha_{1}(\lambda) I\right)=\operatorname{span}\left\{u_{\alpha_{1}(\lambda)}\right\}:=V_{1}$, and $\alpha_{1}(\lambda)$ increases from $\Gamma$ (see (1.3) for the definition of $\Gamma$ ) to $\xi_{1}$ as $\lambda$ increases from $\Gamma$ to $\infty$. 


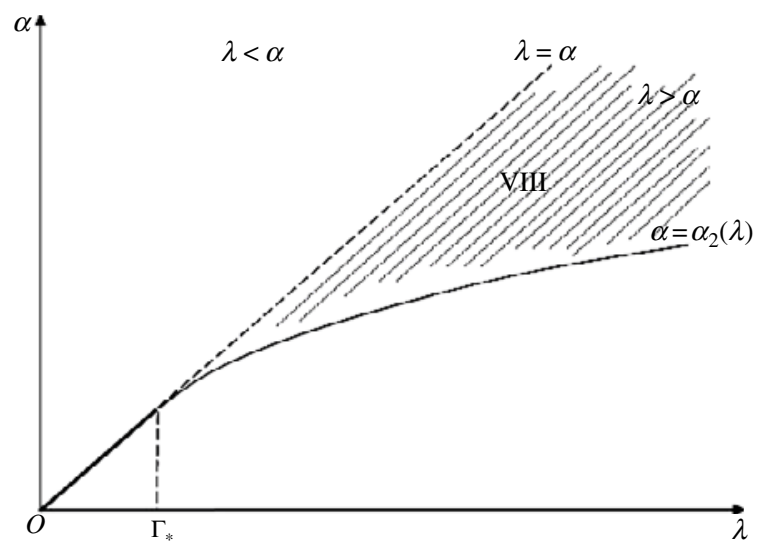

FIGURE 2. Sign-changing solution of Problem (1.1) in the region VIII $\backslash \sigma_{p}\left(L_{\lambda}\right)$.

Define

$$
\Gamma_{*}=\inf _{u \in H^{1}\left(\mathbb{R}^{N}\right) \cap V_{1}^{\perp}} \frac{\int_{\mathbb{R}^{N}}|\nabla u|^{2} d x}{\int_{\mathbb{R}^{N}}(1-g) u^{2} d x} .
$$

Now let $\lambda \in\left(\Gamma_{*}, \infty\right)$ be fixed; we define

$$
\alpha_{2}(\lambda)=\inf \left\{\Phi(u): u \in H^{1}\left(\mathbb{R}^{N}\right) \cap V_{1}^{\perp} \text { and }\|u\|_{2}=1\right\} .
$$

Similar to [11, Proposition 2.1], we can prove that there exists $u \in H^{1}\left(\mathbb{R}^{N}\right) \cap V_{1}^{\perp}$ such that $\Phi(u)$ archives $\alpha_{2}(\lambda)$, then the Lagrange-multipliers method implies that $\alpha_{2}(\lambda)$ is an eigenvalue of $L_{\lambda}$. By the simplicity of $\alpha_{1}(\lambda)$, we obtain that $\alpha_{2}(\lambda)$ is the second eigenvalue of $L_{\lambda}$. Moreover, it is easy to prove that $\alpha_{2}(\lambda)$ is increasing in $\lambda$.

In this paper, by using the variational method, we show that Problem (1.1) has at least a sign-changing solution in the region VIII $\backslash \sigma_{p}\left(L_{\lambda}\right)$ in Figure 2, where $\sigma_{p}\left(L_{\lambda}\right)$ denotes the point spectrum of $L_{\lambda}$.

Now we give the main result of this paper.

THEOREM 1.1. Assume that $f$ satisfies $\left(f_{1}\right)-\left(f_{4}\right)$. If $(\lambda, \alpha)$ is in the region VIII $\backslash$ $\sigma_{p}\left(L_{\lambda}\right)$ in Figure 2, that is, $\alpha>\alpha_{2}(\lambda)$ and $\lambda>\alpha$, moreover, $\alpha \notin \sigma_{p}\left(L_{\lambda}\right)$, then Problem (1.1) has at least a sign-changing solution. In addition, Problem (1.1) has a positive solution and a negative solution.

In the rest of the section, we list some preliminaries which we use later.

Recall that a functional $I$ defined on a Banach space $H$ is said to satisfy the (PS) condition if any sequence $\left\{u_{n}\right\} \subset H$ satisfying $\left|I\left(u_{n}\right)\right| \leq c$ and $I^{\prime}\left(u_{n}\right) \rightarrow 0$ as $n \rightarrow \infty$ possesses a convergent subsequence.

Proposition 1.2 (Liu and Sun [5, Theorem 3.2]). Let $X$ be a Hilbert space and let $f$ be a $C^{1}$ functional defined on $X$. Assume that $f$ satisfies the $(P S)$ condition on $X$ 
and $f^{\prime}(u)$ has the expression $f^{\prime}(u)=u-A u$ for $u \in X$. Assume that $D_{1}$ and $D_{2}$ are open convex subsets of $X$ with the properties that $D_{1} \cap D_{2} \neq \varnothing, A\left(\partial D_{1}\right) \subset D_{1}$ and $A\left(\partial D_{2}\right) \subset D_{2}$. If there exists a path $h:[0,1] \rightarrow X$ such that

$$
h(0) \in D_{1} \backslash D_{2}, \quad h(1) \in D_{2} \backslash D_{1}
$$

and

$$
\inf _{u \in \overline{D_{1}} \cap \overline{D_{2}}} f(u)>\sup _{t \in[0,1]} f(h(t)),
$$

then $f$ has at least four critical points, one in $D_{1} \cap D_{2}$, one in $D_{1} \backslash \overline{D_{2}}$, one in $D_{2} \backslash \overline{D_{1}}$, and one in $X \backslash\left(\overline{D_{1}} \cup \overline{D_{2}}\right)$.

In a given Banach space, $\rightarrow$ and $\rightarrow$ denote the strong convergence and the weak convergence, respectively. Denote $\|u\|_{s}=\left(\int_{\mathbb{R}^{N}}|u|^{s} d x\right)^{1 / s}$. We use

$$
\langle u, v\rangle_{\lambda}=\int_{\mathbb{R}^{N}}\left(\nabla u \nabla v+V_{\lambda}(x) u v\right) d x
$$

as the inner product in the Hilbert space

$$
E_{\lambda}=\left\{u \in H^{1}\left(\mathbb{R}^{N}\right): \int_{\mathbb{R}^{N}} V_{\lambda}(x) u^{2} d x<\infty\right\},
$$

and induced norm $\|u\|_{\lambda}=\sqrt{\langle u, u\rangle_{\lambda}}$. By (G) the norm $\|u\|_{\lambda}$ is equivalent to the norm $\|u\|=\left(\int_{\mathbb{R}^{N}}\left(|\nabla u|^{2}+u^{2}\right) d x\right)^{1 / 2}$.

Finally, $c, c_{0}, c_{1}, c_{2}, \ldots$ denote (possibly different) positive constants.

\section{Proof of Theorem 1.1}

By the assumptions on $V_{\lambda}$ and $f$, the functional $I: E_{\lambda} \rightarrow \mathbb{R}$, corresponding to Problem (1.1), defined by

$$
I(u)=\frac{1}{2}\|u\|_{\lambda}^{2}-\int_{\mathbb{R}^{N}} F(x, u) d x
$$

is continuously (Fréchet) differentiable, where $F(x, u)=\int_{0}^{u} f(x, t) d t$. Therefore, the critical points of the functional $I$ correspond to solutions of Problem (1.1).

Let us write the gradient of $I$ at $u$ by

$$
I^{\prime}(u)=u-A(u), \quad A: E_{\lambda} \rightarrow E_{\lambda}, \quad A(u)=\left(-\Delta+V_{\lambda}\right)^{-1} f(x, u) .
$$

Then $\langle A(u), v\rangle=\int_{\mathbb{R}^{N}} f(x, u) v d x$ for all $v \in E_{\lambda}$. We consider the convex cones $P=\left\{u \in E_{\lambda}: u \geq 0\right\}$ and $-P=\left\{u \in E_{\lambda}: u \leq 0\right\}$. For $\varepsilon>0$, we denote

$$
P_{\varepsilon}=\left\{u \in E_{\lambda}: \operatorname{dist}(u, P)<\varepsilon\right\} \quad \text { and } \quad-P_{\varepsilon}=\left\{u \in E_{\lambda}: \operatorname{dist}(u,-P)<\varepsilon\right\} .
$$

Note that $P_{\varepsilon}$ and $-P_{\varepsilon}$ are open convex subsets of $E_{\lambda}$, therefore $E_{\lambda} \backslash\left(\overline{P_{\varepsilon}} \cup\left(\overline{-P_{\varepsilon}}\right)\right)$ contains only sign-changing functions. 
LEMMA 2.1. The functional I satisfies the (PS) condition.

PROOF. Let $\left\{u_{n}\right\}$ be a sequence in $E_{\lambda}$ such that $\left|I\left(u_{n}\right)\right| \leq c, I^{\prime}\left(u_{n}\right) \rightarrow 0$ as $n \rightarrow \infty$. We first prove that $\left\{u_{n}\right\}$ is bounded in $E_{\lambda}$. In fact, otherwise, we may suppose that $\left\|u_{n}\right\|_{\lambda} \rightarrow \infty$. Set $w_{n}=u_{n} /\left\|u_{n}\right\|_{\lambda}$. Obviously, $\left\{w_{n}\right\}$ is bounded in $E_{\lambda}$. Passing to a subsequence, still denoted by $\left\{w_{n}\right\}$, we may assume that there exists $w \in E_{\lambda}$ such that

$$
\begin{aligned}
w_{n} & \rightarrow w \quad \text { in } E_{\lambda}, \\
w_{n} & \rightarrow w \quad \text { in } L_{\mathrm{loc}}^{s}\left(\mathbb{R}^{N}\right) \text { for } 2 \leq s<2^{*}, \\
w_{n}(x) & \rightarrow w(x) \quad \text { almost everywhere on } \mathbb{R}^{N} .
\end{aligned}
$$

We claim that $w \neq \equiv 0$ in $\mathbb{R}^{N}$. If $w=0$, then $w_{n} \rightarrow 0$ in $L_{\text {loc }}^{2}\left(\mathbb{R}^{N}\right)$. We show that for each $\lambda>0$

$$
\int_{\mathbb{R}^{N}}\left(V_{\lambda}(x)-(1+\lambda)\right) w_{n}^{2} d x \rightarrow 0 \quad \text { as } n \rightarrow \infty .
$$

Indeed, by (G), for every $\varepsilon^{\prime}>0$ there exists $R_{*}>0$ such that $\bar{\Omega} \subset B_{R_{*}}$, and

$$
\left|V_{\lambda}(x)-(1+\lambda)\right|<\varepsilon^{\prime}, \quad x \in \mathbb{R}^{N} \backslash B_{R_{*}} .
$$

So, for all $n \in \mathbb{N}$, we obtain that there exists some positive constant $c_{1}$ such that

$$
\left|\int_{\mathbb{R}^{N} \backslash B_{R_{*}}}\left(V_{\lambda}(x)-(1+\lambda)\right) w_{n}^{2} d x\right| \leq \varepsilon^{\prime}\left\|w_{n}\right\|_{2}^{2} \leq c_{1} \varepsilon^{\prime} .
$$

Also, since $w_{n} \rightarrow 0$ in $L_{\text {loc }}^{2}\left(\mathbb{R}^{N}\right)$ and note that $\left|V_{\lambda}(x)-(1+\lambda)\right| \leq \lambda$, so there exists a constant $c_{2}>0$ such that

$$
\left|\int_{B_{R_{*}}}\left(V_{\lambda}(x)-(1+\lambda)\right) w_{n}^{2} d x\right| \leq \lambda \int_{B_{R_{*}}} w_{n}^{2} d x<c_{2} \varepsilon^{\prime}
$$

for $n$ large enough. Thus,

$$
\begin{aligned}
\int_{\mathbb{R}^{N}}\left(V_{\lambda}(x)-(1+\lambda)\right) w_{n}^{2} d x=\int_{B_{R_{*}}}\left(V_{\lambda}(x)-(1+\lambda)\right) w_{n}^{2} d x \\
\quad+\int_{\mathbb{R}^{N} \backslash B_{R_{*}}}\left(V_{\lambda}(x)-(1+\lambda)\right) w_{n}^{2} d x \rightarrow 0
\end{aligned}
$$

as $n \rightarrow \infty$, which is (2.1). Thus, we have

$$
\lim _{n \rightarrow \infty} \int_{\mathbb{R}^{N}}\left(\left|\nabla w_{n}\right|^{2}+(1+\lambda) w_{n}^{2}\right) d x=\lim _{n \rightarrow \infty}\left\|w_{n}\right\|_{\lambda}^{2}=1
$$

and then

$$
\limsup _{n \rightarrow \infty} \int_{\mathbb{R}^{N}} w_{n}^{2} d x \leq \frac{1}{1+\lambda}
$$


By $\left(f_{1}\right)$ and $\left(f_{3}\right)$, for any $\varepsilon^{\prime}>0$, there exists $M>0$ such that

$$
\frac{F(x, t)}{t^{2}}<\frac{1+\alpha}{2}+\frac{\varepsilon^{\prime}}{4}, \quad \forall|t| \geq M,|x| \leq R
$$

for all $R>0$. Thus,

$$
\begin{aligned}
\int_{\left\{|x| \leq R,\left|u_{n}(x)\right| \geq M\right\}} \frac{F\left(x, u_{n}\right)}{u_{n}^{2}} w_{n}^{2} d x & \leq \frac{1+\alpha}{2} \int_{\mathbb{R}^{N}} w_{n}^{2} d x+\frac{\varepsilon^{\prime}}{4}\left\|w_{n}\right\|_{\lambda}^{2} \\
& =\frac{1+\alpha}{2} \int_{\mathbb{R}^{N}} w_{n}^{2} d x+\frac{\varepsilon^{\prime}}{4}
\end{aligned}
$$

for all $n \in \mathbb{N}$.

It is clear that we may choose $R>0$ large enough such that

$$
\int_{\left\{|x| \geq R,\left|u_{n}(x)\right| \geq M\right\}} \frac{F\left(x, u_{n}\right)}{u_{n}^{2}} w_{n}^{2} d x \leq \frac{\varepsilon^{\prime}}{4}
$$

holds for all $n \in \mathbb{N}$. By $\left(f_{4}\right)$,

$$
\int_{\left\{|x| \geq R,\left|u_{n}(x)\right| \leq M\right\}} \frac{F\left(x, u_{n}\right)}{u_{n}^{2}} w_{n}^{2} d x \leq \sup _{|x| \geq R,\left|u_{n}(x)\right| \leq M} \frac{F\left(x, u_{n}\right)}{u_{n}^{2}}\left\|w_{n}\right\|_{\lambda}^{2} \leq \frac{\varepsilon^{\prime}}{4}
$$

for all $n \in \mathbb{N}$. Note that $w_{n} \rightarrow 0$ in $L_{\text {loc }}^{2}\left(\mathbb{R}^{N}\right)$ as $n \rightarrow \infty$. Then

$$
\int_{\left\{|x| \leq R,\left|u_{n}(x)\right| \leq M\right\}} \frac{F\left(x, u_{n}\right)}{u_{n}^{2}} w_{n}^{2} d x \leq \frac{\varepsilon^{\prime}}{4}
$$

for $n$ large enough. Combining (2.3)-(2.6) we conclude that for $n$ large enough

$$
\int_{\mathbb{R}^{N}} \frac{F\left(x, u_{n}\right)}{u_{n}^{2}} w_{n}^{2} d x \leq \frac{1+\alpha}{2} \int_{\mathbb{R}^{N}} w_{n}^{2} d x+\varepsilon^{\prime} .
$$

Then by $\left|I\left(u_{n}\right)\right| \leq c,(2.2)$ and (2.7), we know that

$$
\frac{1}{2}=\lim _{n \rightarrow \infty} \int_{\mathbb{R}^{N}} \frac{F\left(x, u_{n}\right)}{u_{n}^{2}} w_{n}^{2} d x \leq \frac{1+\alpha}{2(1+\lambda)},
$$

which is impossible since $\lambda>\alpha$. So, we have shown that $w \not \equiv 0$.

By $I^{\prime}\left(u_{n}\right) \rightarrow 0$ as $n \rightarrow \infty$, for all $\varphi \in E_{\lambda}$

$$
\left\langle I^{\prime}\left(u_{n}\right), \varphi\right\rangle=\int_{\mathbb{R}^{N}}\left(\nabla u_{n} \nabla \varphi+V_{\lambda}(x) u_{n} \varphi-f\left(x, u_{n}\right) \varphi\right) d x \rightarrow 0
$$

as $n \rightarrow \infty$. Dividing (2.8) by $\left\|u_{n}\right\|_{\lambda}$ we have

$$
\int_{\mathbb{R}^{N}}\left(\nabla w_{n} \nabla \varphi+V_{\lambda}(x) w_{n} \varphi-\frac{f\left(x, u_{n}\right)}{u_{n}} w_{n} \varphi\right) d x=o(1) .
$$


Since $u_{n}(x)=\left\|u_{n}\right\|_{\lambda} w_{n}(x) \rightarrow \infty$ for $x \notin\left\{x \in \mathbb{R}^{N} \mid w(x)=0\right\}, \quad$ and $\quad w_{n}(x) \rightarrow$ $w(x)$ almost everywhere in $\mathbb{R}^{N}$, it implies by $\left(f_{3}\right)$ that

$$
\frac{f\left(x, u_{n}(x)\right)}{u_{n}(x)} w_{n}(x) \rightarrow(1+\alpha) w(x) \quad \text { almost everywhere in } \mathbb{R}^{N} .
$$

Also by $\left(f_{3}\right)$, we know that $f(x, t) / t$ is bounded on $\mathbb{R}^{N} \times(\mathbb{R} \backslash\{0\})$. Then the sequence $\left\{\left(f\left(x, u_{n}(x)\right) / u_{n}(x)\right) w_{n}(x)\right\}$ is bounded in $L^{2}\left(\mathbb{R}^{N}\right)$, thus there exists a subsequence, still denoted by the same subscripts, such that

$$
\frac{f\left(x, u_{n}\right)}{u_{n}} w_{n} \rightarrow(1+\alpha) w \text { in } L^{2}\left(\mathbb{R}^{N}\right) .
$$

Therefore, by (2.9) and the weak convergence of $\left\{w_{n}\right\}$ in $E_{\lambda}$, we obtain

$$
\int_{\mathbb{R}^{N}}\left(\nabla w \nabla \varphi+V_{\lambda}(x) w \varphi-(1+\alpha) w \varphi\right) d x=0,
$$

that is,

$$
\int_{\mathbb{R}^{N}}(\nabla w \nabla \varphi+\lambda g(x) w \varphi) d x=\alpha \int_{\mathbb{R}^{N}} w \varphi d x .
$$

From this we see that $w$ satisfies

$$
-\Delta w+\lambda g(x) w=\alpha w .
$$

This is impossible since $\alpha \notin \sigma_{p}\left(L_{\lambda}\right)$. Therefore, $\left\{u_{n}\right\}$ is bounded in $E_{\lambda}$.

Next we prove that there exists $u \in E_{\lambda}$ such that $\left\|u_{n}\right\|_{\lambda} \rightarrow\|u\|_{\lambda}$ as $n \rightarrow \infty$.

Indeed, by the boundedness of $\left\{u_{n}\right\}$, passing to a subsequence, we may assume that $u_{n} \rightarrow u$ in $E_{\lambda}$ and $u_{n} \rightarrow u$ in $L_{\text {loc }}^{s}\left(\mathbb{R}^{N}\right)$. Since $\left\langle I^{\prime}\left(u_{n}\right), \varphi\right\rangle \rightarrow 0$ as $n \rightarrow \infty$, we let $\varphi=u_{n}-u$ and obtain

$$
\begin{aligned}
0 & \leq \limsup _{n \rightarrow \infty}\left(\left\|u_{n}\right\|_{\lambda}^{2}-\|u\|_{\lambda}^{2}\right)=\limsup _{n \rightarrow \infty}\left\langle u_{n}, u_{n}-u\right\rangle \\
& =\limsup _{n \rightarrow \infty} \int_{\mathbb{R}^{N}} f\left(x, u_{n}\right)\left(u_{n}-u\right) d x .
\end{aligned}
$$

Clearly, by $\left(f_{3}\right), \lim _{|t| \rightarrow \infty} f(x, t) / t^{p-1}=0$ for all fixed $p \in\left(2,2^{*}\right)$. Then it follows from $\left(f_{1}\right),\left(f_{2}\right)$ that for each $\varepsilon^{\prime}>0$ there exists $C_{\varepsilon^{\prime}}>0$ such that

$$
|f(x, t)| \leq \varepsilon^{\prime}|t|+C_{\varepsilon^{\prime}}|t|^{p-1} .
$$

By (2.11) and the Hölder inequality, for $r \geq 1$

$$
\begin{aligned}
\int_{\left\{\left|u_{n}(x)\right| \geq r\right\}} f\left(x, u_{n}\right)\left(u_{n}-u\right) d x & \leq 2 C \int_{\left\{\left|u_{n}(x)\right| \geq r\right\}}\left|u_{n}\right|^{p-1}\left|u_{n}-u\right| d x \\
& =2 C \int_{\left\{\left|u_{n}(x)\right| \geq r\right\}} \frac{1}{\left|u_{n}\right|^{2^{*}-p}}\left|u_{n}\right|^{2^{*}-1}\left|u_{n}-u\right| d x \\
& \leq 2 C r^{p-2^{*}}\left\|u_{n}\right\|_{2^{*}}^{2^{*}-1}\left\|u_{n}-u\right\|_{2^{*}},
\end{aligned}
$$


where $C>0$ is a constant. Since $p<2^{*}$, we may choose $r$ so large that for all $n \in \mathbb{N}$

$$
\int_{\left\{\left|u_{n}(x)\right| \geq r\right\}} f\left(x, u_{n}\right)\left(u_{n}-u\right) d x \leq \frac{\varepsilon^{\prime}}{3} .
$$

Moreover, by $\left(f_{4}\right)$, there exists $R>0$ such that for all $n \in \mathbb{N}$

$$
\int_{\left\{|x| \geq R,\left|u_{n}(x)\right| \leq r\right\}} f\left(x, u_{n}\right)\left(u_{n}-u\right) d x \leq \frac{\varepsilon^{\prime}}{3} .
$$

This implies by (2.11), the Hölder inequality and the compactness of the embedding $H^{1}\left(\mathbb{R}^{N}\right) \hookrightarrow L_{\text {loc }}^{s}\left(\mathbb{R}^{N}\right)$ that for $n$ large enough

$$
\int_{\left\{|x| \leq R,\left|u_{n}(x)\right| \leq r\right\}} f\left(x, u_{n}\right)\left(u_{n}-u\right) d x \leq \frac{\varepsilon^{\prime}}{3},
$$

so by (2.12)-(2.14)

$$
\int_{\mathbb{R}^{N}} f\left(x, u_{n}\right)\left(u_{n}-u\right) d x \leq \varepsilon^{\prime}
$$

for $n$ large enough. From this and (2.10) we know that $\left\|u_{n}\right\|_{\lambda} \rightarrow\|u\|_{\lambda}$.

Finally, the locally uniform convexity of $E_{\lambda}$ gives that $u_{n} \rightarrow u$ in $E_{\lambda}$ as $n \rightarrow \infty$. The lemma is proved.

LEMMA 2.2. Assume that $\left(f_{1}\right)-\left(f_{3}\right)$ hold, then there exists $\varepsilon_{0}>0$ such that for all $\varepsilon: 0<\varepsilon \leq \varepsilon_{0}$ there holds

$$
A\left(\partial\left( \pm P_{\varepsilon}\right)\right) \subset \pm P_{\varepsilon}
$$

Moreover, if $u \in \pm P_{\varepsilon}$ is a solution of Problem (1.1), then $u \in \pm P$.

PRoOF. Indeed, if $u \in E_{\lambda}$ and $u^{+}=\max \{u, 0\}, u^{-}=\min \{u, 0\}$, then

$$
\operatorname{dist}(A(u), P)=\min _{w \in P}\|A(u)-w\|_{\lambda}=\min _{w \in P}\left\|A(u)^{+}+A(u)^{-}-w\right\|_{\lambda} \leq\left\|A(u)^{-}\right\|_{\lambda}
$$

Let $s \in\left[2,2^{*}\right)$, then by the continuity of the embedding $E_{\lambda} \hookrightarrow L^{s}\left(\mathbb{R}^{N}\right)$, there exists $C_{s}>0$ such that

$$
\left\|u^{\mp}\right\|_{s}=\min _{w \in \pm P}\|u-w\|_{s} \leq C_{s} \min _{w \in \pm P}\|u-w\|_{\lambda}=C_{s} \operatorname{dist}(u, \pm P)
$$

We claim that $A(u) \in P_{\varepsilon}$ for any $u \in \partial P_{\varepsilon}$. 
In fact, it follows from $(2.11),(2.15),\left(f_{1}\right)$ and the Hölder inequality that

$$
\begin{aligned}
\operatorname{dist}(A(u), P)\left\|A(u)^{-}\right\|_{\lambda} & \leq\left\|A(u)^{-}\right\|_{\lambda}^{2}=\left\langle A(u), A(u)^{-}\right\rangle \\
& =\int_{\mathbb{R}^{N}} f(x, u) A(u)^{-} d x \\
& \leq \int_{\{u \geq 0\}} f(x, u) A(u)^{-} d x+\int_{\{u \leq 0\}} f(x, u) A(u)^{-} d x d x \\
& \leq \int_{\{u \leq 0\}} f\left(x, u^{-}\right) A(u)^{-} d x \\
& \leq \int_{\{u \leq 0\}}\left(\varepsilon^{\prime}\left|u^{-}\right|+C_{\varepsilon^{\prime}}\left|u^{-}\right|^{p-1}\right) A(u)^{-} d x \\
& \leq \varepsilon^{\prime} \tilde{c} \operatorname{dist}(u, P)\left\|A(u)^{-}\right\|_{\lambda}+\tilde{C}_{\varepsilon^{\prime}, p} \operatorname{dist}(u, P)^{p-1}\left\|A(u)^{-}\right\|_{\lambda} .
\end{aligned}
$$

Taking $\varepsilon^{\prime}=1 / 2 \tilde{c}$, then we obtain

$$
\operatorname{dist}(A(u), P) \leq \frac{1}{2} \operatorname{dist}(u, P)+\tilde{C} \operatorname{dist}(u, P)^{p-1},
$$

where $\tilde{C}>0$ is a constant. Let $\varepsilon_{0}=(1 / 4 \tilde{C})^{1 /(p-2)}$, then for all $\varepsilon$ with $0<\varepsilon \leq \varepsilon_{0}$, we have

$$
\operatorname{dist}(A(u), P) \leq \frac{3}{4} \operatorname{dist}(u, P)
$$

for all $u \in P_{\varepsilon}$. Clearly, $\operatorname{dist}(A(u), P) \leq \frac{3}{4} \varepsilon<\varepsilon$ for every $u \in \partial P_{\varepsilon}$, that is, $A(u) \in P_{\varepsilon}$ for all $u \in \partial P_{\varepsilon}$. Hence, $A\left(\partial P_{\varepsilon}\right) \subset P_{\varepsilon}$. In a similar way, $A\left(\partial\left(-P_{\varepsilon}\right)\right) \subset-P_{\varepsilon}$. If $u \in P_{\varepsilon}$ is a solution of Problem (1.1), then $I^{\prime}(u)=u-A(u)=0$, that is, $u=A(u)$, by (2.16), then $u \in P$. Similarly, if $u \in-P_{\varepsilon}$, then $u \in-P$.

Lemma 2.3. Assume $\left(f_{1}\right)-\left(f_{3}\right)$ hold. Let $0<\varepsilon \leq \varepsilon_{0}$, then there exists $C_{*}>-\infty$ such that $\inf _{\overline{P_{\varepsilon}} \cap\left(-\overline{P_{\varepsilon}}\right)} I(u)=C_{*}$.

PROOF. It follows from (2.11) that

$$
\begin{aligned}
I(u) & =\frac{1}{2}\|u\|_{\lambda}^{2}-\int_{\mathbb{R}^{N}} F(x, u) d x \\
& \geq \frac{1}{2}\|u\|_{\lambda}^{2}-\frac{1}{2} \varepsilon^{\prime} \int_{\mathbb{R}^{N}} u^{2} d x-C_{\varepsilon^{\prime}, p} \int_{\mathbb{R}^{N}}|u|^{p} d x \\
& \geq-\frac{1}{2} \varepsilon^{\prime}\|u\|_{2}^{2}-C_{\varepsilon^{\prime}, p}\|u\|_{p}^{p} .
\end{aligned}
$$

By (2.15) we have $\left\|u^{ \pm}\right\|_{s} \leq C_{s} \operatorname{dist}(u, \mp P) \leq C_{s} \varepsilon_{0}$ for every $u \in P_{\varepsilon} \cap\left(-P_{\varepsilon}\right)$. So there exists $C_{*}>-\infty$ such that $\inf _{\overline{P_{\varepsilon}} \cap\left(-\overline{P_{\varepsilon}}\right)} I(u)=C_{*}$. Hence, the lemma is proved. 
PROOF OF THEOREM 1.1. Let $u_{\alpha_{2}(\lambda)}$ be an eigenfunction corresponding to the eigenvalue $\alpha_{2}(\lambda)$, and set $W:=\left\{u \in E_{\lambda}:\|u\|_{\lambda}=1, u \in \operatorname{span}\left\{u_{\alpha_{1}(\lambda)}, u_{\alpha_{2}(\lambda)}\right\}\right\}$. Define a path $\gamma:[0,1] \rightarrow W$

$$
\gamma(t)=\cos (\pi t) u_{\alpha_{1}(\lambda)}+\sin (\pi t) u_{\alpha_{2}(\lambda)}
$$

connecting $\gamma(0)=u_{\alpha_{1}(\lambda)}$ and $\gamma(1)=-u_{\alpha_{1}(\lambda)}$. Now let $h_{R}(t)=R \gamma(t)$. Since $0 \leq$ $F(x, t) / t^{2} \leq C$ for all $t \in \mathbb{R}, x \in \mathbb{R}^{N}$, and

$$
\lim _{R \rightarrow+\infty} \frac{F(x, R \gamma(t))}{R^{2} \gamma(t)^{2}}=\frac{1+\alpha}{2} \text { for almost every } x \in \mathbb{R}^{N},
$$

thus, it follows by Lebesgue's theorem that

$$
\begin{aligned}
\lim _{R \rightarrow+\infty} \frac{I\left(h_{R}(t)\right)}{R^{2}}= & \frac{1}{2} \int_{\mathbb{R}^{N}}\left(|\nabla \gamma(t)|^{2}+\gamma(t)^{2}+\lambda g(x) \gamma(t)^{2}\right) d x \\
& -\lim _{R \rightarrow+\infty} \int_{\mathbb{R}^{N}} \frac{F(x, R \gamma(t))}{R^{2} \gamma(t)^{2}} \gamma(t)^{2} d x \\
= & \frac{1}{2}-\frac{1}{2}(1+\alpha) \int_{\mathbb{R}^{N}}\left(\cos ^{2}(\pi t) u_{\alpha_{1}(\lambda)}^{2}+\sin ^{2}(\pi t) u_{\alpha_{2}(\lambda)}^{2}\right) d x \\
= & \frac{1}{2}-\frac{1}{2}(1+\alpha)\left(\frac{\cos ^{2}(\pi t)}{\alpha_{1}(\lambda)+1}+\frac{\sin ^{2}(\pi t)}{\alpha_{2}(\lambda)+1}\right) \\
& \frac{1}{2}\left(1-\frac{1+\alpha}{1+\alpha_{2}(\lambda)}\right)<0 .
\end{aligned}
$$

So, this yields that there exists $R_{0}$ such that $I\left(h_{R_{0}}(t)\right)<C_{*}-1$. Hence, we obtain

$$
\inf _{\overline{P_{\varepsilon}} \cap\left(-\overline{P_{\varepsilon}}\right)} I(u)>\sup _{t \in[0,1]} I\left(h_{R_{0}}(t)\right) .
$$

Obviously $h_{R_{0}}(0) \in P_{\varepsilon} \backslash\left(-P_{\varepsilon}\right), h_{R_{0}}(1) \in\left(-P_{\varepsilon}\right) \backslash P_{\varepsilon}$. By using Lemmas $2.1-2.3$ and Proposition 1.2, we can find a critical point in $E_{\lambda} \backslash\left(\overline{P_{\varepsilon}} \cup\left(\overline{-P_{\varepsilon}}\right)\right)$, which is a signchanging solution of Problem (1.1). Also we have a critical point in $P_{\varepsilon} \backslash\left(\overline{-P_{\varepsilon}}\right)$ and a critical point in $\left(-P_{\varepsilon}\right) \backslash \overline{P_{\varepsilon}}$, which correspond to a positive solution and a negative solution of Problem (1.1).

\section{Acknowledgements}

The first author expresses her sincere thanks to Professors J. Q. Liu and C. A. Stuart for their fruitful discussions and encouragement.

\section{References}

[1] T. Bartsch, Z. L. Liu and T. Weth, 'Sign changing solutions of superlinear Schrödinger equations', Comm. Partial Differential Equations 29 (2004), 25-42.

[2] T. Bartsch, A. Pankov and Z. Q. Wang, 'Nonlinear Schrödinger equations with steep potential well', Comm. Contemp. Math. 3 (2001), 1-21. 
[3] Y. H. Ding and K. Tanaka, 'Multiplicity of positive solutions of a nonlinear Schrödinger equation', Manuscripta Math. 112 (2003), 109-135.

[4] Y. Q. Li, Z. Q. Wang and J. Zeng, 'Ground states of nonlinear Schrödinger equations with potentials', Ann. Inst. H. Poincaré Anal. Non Linéaire 23 (2006), 829-837.

[5] Z. L. Liu and J. X. Sun, 'Invariant sets of descending flow in critical point theory with applications to nonlinear differential equations', J. Differential Equations 172 (2001), 257-299.

[6] C. A. Stuart, 'An introduction to elliptic equations on $\mathbb{R}^{N}$, in: Nonlinear Functional Analysis and Applications to Differential Equations (eds. A. Ambrosetti, K. C. Chang and I. Ekeland) (World Scientific, Singapore, 1998).

[7] C. A. Stuart and H. S. Zhou, 'Positive eigenfunctions of a Schrödinger operator', J. London Math. Soc. 72 (2005), 429-441.

[8] - 'Global branch of solutions for nonlinear Schrödinger equations with deepening potential well', Proc. London Math. Soc. 92 (2006), 655-681.

[9] H. Tehrani, 'Existence results for an indefinite unbounded perturbation of a resonant Schrödinger equation', J. Differential Equations 236 (2007), 1-28.

[10] F. A. van Heerden and Z. Q. Wang, 'Schrödinger type equation with asymptotically linear nonlinearities', Differential Integral Equations 16 (2003), 257-280.

[11] Z. P. Wang and H. S. Zhou, 'Positive solution for nonlinear Schrödinger equation with deepening potential well', J. Eur. Math. Soc. 11(3) (2009), 545-573.

XIANGQING LIU, Department of Mathematics, Soochow University, Suzhou 215006, P.R. China

and

Department of Mathematics, Yunnan Normal University, Kunming 650092, P.R. China e-mail: lxq8u8@163.com

YISHENG HUANG, Department of Mathematics, Soochow University,

Suzhou 215006, P.R. China

e-mail: yishengh@suda.edu.cn 